\title{
Prediction of chronic kidney disease after orthotopic liver transplantation: development and validation of a nomogram model
}

Dandan Guo, Huifang Wang, Jun Liu, Hang Liu, Ming Zhang, Zixuan Fu and Xuemei Liu*

\begin{abstract}
Background: We aimed to develop and validate a nomogram model for predicting CKD after orthotopic liver transplantation (OLT).

Methods: The retrospective data of 399 patients who underwent transplantation and were followed in our centre were collected. They were randomly assigned to the training set $(n=293)$ and validation set $(n=106)$. Multivariable Cox regression analysis was performed in the training set to identify predictors of CKD. According to the Cox regression analysis results, a nomogram model was developed and validated. The renal function of recipients was monitored, and the long-term survival prognosis was assessed.
\end{abstract}

Results: The incidence of CKD at 5 years after OLT was 25.6\%. Cox regression analysis identified several predictors of post-OLT CKD, including recipient age at surgery (HR 1.036,95\% Cl 1.006-1.068; $p=0.018)$, female sex (HR 2.867, 95\% Cl 1.709-4.810; $p<0.001$ ), preoperative hypertension (HR 1.670,95\% Cl 0.962-2.898; $p=0.068$ ), preoperative eGFR (HR $0.996,95 \%$ Cl 0.991-1.001; $p=0.143)$, uric acid at 3 months (HR 1.002, 95\% Cl 1.001-1.004; $p=0.028)$, haemoglobin at 3 months (HR 0.970,95\% Cl 0.956-0.983; $p<0.001)$, and average concentration of cyclosporine A at 3 months (HR $1.002,95 \% \mathrm{Cl} 1.001-1.003 ; p<0.001)$. According to these parameters, a nomogram model for predicting CKD after OLT was constructed and validated. The C-indices were 0.75 and 0.80 in the training and validation sets. The calibration curve of the nomogram showed that the CKD probabilities predicted by the nomogram agreed with the observed probabilities at 1,3 , and 5 years after OLT $(p>0.05)$. Renal function declined slowly year by year, and there were significant differences between patients divided by these predictors. Kaplan-Meier survival analysis showed that the survival prognosis of recipients decreased significantly with the progression of renal function.

Conclusions: With excellent predictive abilities, the nomogram may be a simple and reliable tool to identify patients at high risk for CKD and poor long-term prognosis after OLT.

Keywords: Chronic kidney disease, Orthotopic liver transplantation, Nomogram model, Prognosis

*Correspondence: liuxuemei201718@163.com

Department of Nephrology, The Affiliated Hospital of Qingdao University,

No. 16 Jiangsu Road, Qingdao 266003, Shandong, China

\section{Introduction}

Chronic kidney disease (CKD) is a common and significant complication after orthotopic liver transplantation (OLT). With the progression of surgical technology, perioperative treatment, and immunosuppressants, the survival rates of patients after OLT have dramatically 
improved to 90 and $75 \%$ at 1 and 5 years after OLT, respectively [1]. Long-term complications, such as CKD and cardiovascular events, are receiving increasing attention from clinical specialists. The prevalence of CKD reported in the literature was $4 \sim 27.5 \%$ in 1year [2-4], $15 \sim 60 \%$ in 5 years $[2,4,5]$, and $25 \sim 50 \%$ in 10 years after OLT $[6,7]$. CKD is a primary contributor to morbidity and mortality after OLT, including an increased risk of allograft dysfunction, cardiovascular events [8], and death $[4,9]$, which has elicited concern regarding post-OLT management. However, only a limited number of studies have assessed long-term renal outcomes after OLT, which has important implications for patient management.

Studies have shown that the causes of CKD after OLT are complex and may include preoperative (age, female sex, history of diabetes mellitus (DM) or hypertension, hyperlipidaemia, and hepatitis $\mathrm{C}$ ) and postoperative (calcineurin inhibitor (CNI) toxicity, prolonged ischaemia, and haemodynamic instability) factors [6, 10-13]. There have been controversial results regarding a direct association between perioperative AKI and post-OLT CKD $[14,15]$.

A credible and helpful predictive model to identify which patients are at high risk of developing CKD in clinical practice has not been developed, although it would be a useful tool for designing preventative measures, such as early decreased doses or withdrawal of CNIs and administration of non-nephrotoxic immunosuppressants. In this study, we aimed to develop and validate a reliable nomogram model for predicting CKD after OLT. With this model, we can identify patients at high risk for CKD early and take targeted measures to prevent or slow the development and progression of CKD.

\section{Methods}

\section{Study population}

All adult patients who underwent OLT from April 2013 to October 2020 at our centre were enrolled in our study, and their medical records were retrospectively reviewed. Recipients with a minimal follow-up time of 6 months after transplantation were included. The exclusion criteria included retransplantation, baseline estimated glomerular filtration rate $(e G F R)<60 \mathrm{ml} / \mathrm{min} / 1.73 \mathrm{~m}^{2}$, renal replacement therapy (RRT) before liver transplantation, multiorgan transplantation, follow-up less than 6 months, and age $<18$ years. Finally, 399 patients were enrolled in our study. They were randomly allocated to the training set $(n=293)$ and the validation set $(n=106)$ (Fig. 1$)$.

The study was conducted according to the principles of the Declaration of Helsinki and approved by the Medical Ethics Committee of The Affiliated Hospital of Qingdao
University (the ethics approval number is QYFY WZLL 26283).

\section{Data collection and definitions}

Demographic details, relevant preoperative and postoperative laboratory test results, and concentrations of CNIs were recorded. AKI was defined as an increase in serum creatinine $(\mathrm{sCr})$ of at least $26.5 \mu \mathrm{mol} / \mathrm{l}$ within $48 \mathrm{~h}$ or more than $50 \%$ within 7 days after transplantation. Peak aspartate aminotransferase (AST) within the first $24 \mathrm{~h}$ after transplantation was used as a measure of ischaemia-reperfusion injury (IRI) [16]. New-onset CKD was defined as CKD that occurs after OLT and as eGFR $<60 \mathrm{ml} / \mathrm{min} / 1.73 \mathrm{~m}^{2}$ for 3 months, regardless of the presence or absence of structural kidney damage [9]. $\mathrm{sCr}$, eGFR, and CNI levels were recorded before OLT as well as 1-7 days and 1, 3, 6, and 12 months after OLT and yearly thereafter until the end of follow-up. eGFR was calculated according to the Modification of Diet in Renal Disease (MDRD) equation: eGFR $=186 \times$ creatinine $(\mathrm{mg} / \mathrm{dL})^{-1.154} \times(\mathrm{age})^{-0.203} \times 1.212$ (if black) $\times 0.742$ (if female) [17].

According to the Kidney Disease: Improving Global Outcomes (KDIGO) CKD Work Group, the patients' renal function was classified as follows [18]: mildly decreased: GFR $60-89 \mathrm{ml} / \mathrm{min} / 1.73 \mathrm{~m}^{2}$; mildly to severely decreased: GFR $30-59 \mathrm{ml} / \mathrm{min} / 1.73 \mathrm{~m}^{2}$, severely decreased: GFR $15-29 \mathrm{ml} / \mathrm{min} / 1.73 \mathrm{~m}^{2}$; and kidney failure: GFR $<15 \mathrm{ml} / \mathrm{min} / 1.73 \mathrm{~m}^{2}$ [19].

\section{Immunosuppression}

The standard immunosuppressive regimen after OLT consisted of CNIs combined with mycophenolate mofetil (MMF) and corticosteroids. Most patients were treated with a triple-drug regimen of tacrolimus (FK506) or cyclosporine A (CsA), MMF, and corticosteroids. Methylprednisolone was given on the day of transplantation, followed by tapering, and then converted to methylprednisolone for oral administration, which was gradually reduced. When renal function was impaired, the introduction of CNIs was delayed. Some patients were switched to mammalian target of rapamycin (mTOR) inhibitor therapy when plasma FK506 failed to reach target levels or if CKD developed. The dose of immunosuppressant was adjusted according to blood concentration.

\section{Statistical analysis}

$\mathrm{R}$ version 4.0.3 ( $\mathrm{R}$ Foundation for Statistical Computing), STATA 15.0 (StataCorp, Texas, USA), and SPSS 25.0 were used to analyse the data. The median and interquartile range (IQR) were used to describe continuous variables, and the Mann-Whitney $U$ test was performed to 


\section{1 patients who received OLT between April 2013 to October 2020}

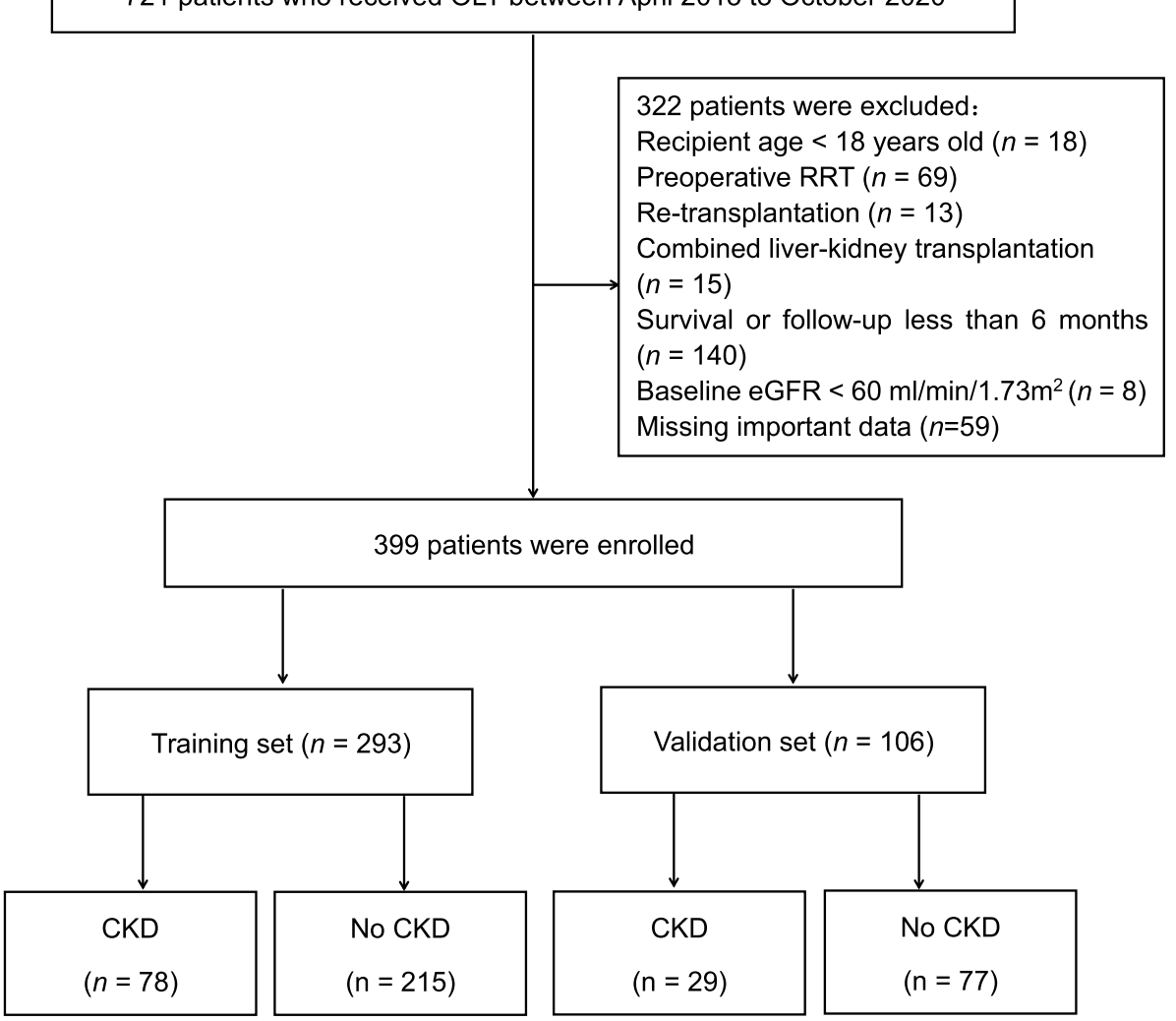

Fig. 1 Flow diagram of patient enrolment

compare parametric variables. Categorical variables were expressed as quantities and percentages, and comparisons were made using the chi-square test or Fisher's exact test. A Cox proportional hazards model was implemented in the training set to identify predictors of the development of CKD after liver transplantation. Factors with $p<0.05$ in the univariable Cox analysis and several factors with clinical significance in previous studies were enrolled in the multivariable Cox analysis. The final Cox regression model was chosen by using forward stepwise regression with the Akaike information criterion (AIC) as the stopping rule. The AIC value for the final model was minimized with the fewest number of variables [20]. For further analysis, a nomogram was constructed based on the results of the Cox regression analysis. Then, we validated the predictive abilities of this model by examining its discrimination and calibration in the training set and validation set. The discrimination was quantified by the $\mathrm{C}$-index, and the calibration was evaluated by the calibration curve. Kaplan-Meier curve analysis with the log-rank test was performed to evaluate the survival time of patients with renal function in different categories. The "rms" package was used for the nomogram and calibration curve. The "survminer" and "survival" packages were used for Kaplan-Meier analysis. For all statistical analyses, $p$-values of $<0.05$ were considered statistically significant.

\section{Results}

\section{Characteristics of the population}

A total of 399 patients were enrolled in our study with a median follow-up of 31.5 months (IQR 18.3-51.6 months), of whom 293 patients were allocated to the training set and 106 patients were allocated to the validation set. The median age at transplantation of all patients was 52 years (IQR 45-59years), and the majority of recipients were males (82.9\%). The main indications for OLT at our centre were hepatitis B virus (HBV)-associated hepatocellular carcinoma (49.1\%), followed by HBV-associated cirrhosis (27.5\%). During the first week after liver transplantation, $71.1 \%$ of the recipients developed AKI, and $36.8 \%$ developed severe AKI (stage 2-3).

\section{Incidence of CKD after OLT}

During the first week after OLT, $44.4 \%$ of recipients experienced a significant decrease in eGFR of 
more than $50 \%$, including $8.3 \%$ of patients who had an eGFR of less than $30 \mathrm{ml} / \mathrm{min} / 1.73 \mathrm{~m}^{2}$. At 1 year, $13.0 \%$ of recipients had eGFR $<60 \mathrm{ml} / \mathrm{min} / 1.73 \mathrm{~m}^{2}$, and $0.8 \%$ had eGFR $<30 \mathrm{ml} / \mathrm{min} / 1.73 \mathrm{~m}^{2}$. The incidences of CKD at 1,3 , and 5 years after OLT were 11.0, 20.3, and 25.6\%, respectively. During the entire follow-up period, $28.6 \%$ of the recipients had renal function decreaesd, and the majority (55.1\%) had eGFR mildly decreased. The mildly to severely decreased eGFR, severely decreased eGFR and endstage renal disease (ESRD) (eGFR $<15 \mathrm{ml} / \mathrm{min} / 1.73 \mathrm{~m}^{2}$ or long-term RRT) were observed in $26.8,1.5$, and $0.5 \%$ of the recipients, respectively. Only one recipient received a kidney transplant 19 months after OLT at our centre.

\section{Cox regression analysis of predictors associated with the development of CKD after OLT}

The incidences of CKD were 26.6 and $27.3 \%$ in the training set and validation set, respectively $(p>0.05)$. Except for several factors with $p<0.05$, including $\mathrm{sCr}$, eGFR before OLT, serum albumin (ALB), and platelet-to-lymphocyte ratio (PLR) at 3 months after OLT, there were no significant differences between the training set and validation set regarding the demographic details, relevant preoperative and postoperative laboratory test results, and concentrations of CNIs $(p>0.05)$ (Tables 1,2 , and 3$)$. The factors with $p<0.05$ in the univariable Cox analysis and several factors with clinical significance in previous studies were included in the multivariable Cox regression model. The multivariable Cox regression analysis identified several recipient predictors associated with post-OLT CKD, including recipient age at surgery (hazard ratio [HR] 1.036, 95\% confidence interval [CI] 1.006-1.068; $p=0.018$ ), female sex (HR 2.867, 95\% CI 1.709-4.810; $p<0.001$ ), preoperative hypertension (HR 1.670, 95\% CI 0.962-2.898; $p=0.068$ ), preoperative eGFR (HR 0.996, 95\% CI 0.991-1.001; $p=0.143$ ), uric acid (UA) at 3 months (HR 1.002, 95\% CI 1.0011.004; $p=0.028)$, haemoglobin $(\mathrm{Hb})$ at 3 months (HR 0.970, 95\% CI 0.956-0.983; $p<0.001$ ), and average concentration of CsA at 3 months after OLT (HR 1.002, 95\% CI 1.001-1.003; $p<0.001$ ) (Table 4). Postoperative AKI was associated with the development of CKD only in the univariable Cox regression analysis. The impact of FK506 was also investigated, and its median levels at 1 and 3 months after OLT were evaluated. Interestingly, these levels were lower in patients who developed CKD (at 1 month CKD 6.6, IQR 4.9$8.5 \mu \mathrm{g} / \mathrm{L}$; no CKD 8.3, IQR $6.4-10.6 \mu \mathrm{g} / \mathrm{L} ; p<0.001$; at 3 months CKD 6.3, IQR 3.5-7.8 $\mu \mathrm{g} / \mathrm{L}$; no CKD 6.6, IQR $4.8-8.4 \mu \mathrm{g} / \mathrm{L} ; p=0.084)$.

\section{Development and validation of a nomogram model} for predicting CKD after OLT

A nomogram model for predicting CKD after OLT was constructed based on the results of the multivariable Cox regression model (Fig. 2). Points were assigned to the seven identified predictors based on their regression coefficients. For an individual patient, the points of each factor can be summed to calculate the estimated probabilities of CKD at 1,3, and 5 years after OLT. Then, the nomogram was validated in the training set and validation set. The C-indices were 0.75 (95\% CI 0.72-0.78) and 0.80 (95\% CI 0.75-0.85) in the training set and validation set, respectively. The calibration curve of the nomogram showed that the CKD probabilities predicted by the nomogram agreed with the observed probabilities at 1,3 , and 5 years after OLT $(p>0.05)$ (Fig. 3). These results indicated that the nomogram model could accurately predict the risk of CKD after OLT using the perioperative predictors mentioned above.

\section{Renal recovery and long-term prognosis of recipients}

After the first week, renal function recovered rapidly in most recipients but decreased slowly after surgery. Based on the predictors of CKD in the multivariable analysis, including recipients' age, sex, hypertension, preoperative eGFR, uric acid, and haemoglobin at 3 months after OLT, we divided the recipients into different groups. The differences in the changes in renal function between these groups are presented in Fig. 4. eGFR dropped sharply in the first 7 days after OLT due to postoperative AKI. Subsequently, eGFR increased sharply as renal function recovered and gradually decreased year by year. Because there were only a few patients with ESRD, they were included in the severely decreased renal function group. After a median follow-up period of 31.5 months (IQR 18.3-51.6 months), the mortality rates were 2.2, 4.1, and $1.8 \%$ in the mildly, mildly to severely, and severely decreased renal function groups, respectively $(p<0.001)$. Kaplan-Meier survival analysis with the log-rank test showed that survival was significantly different among these three groups (log-rank $=70.829, p<0.01$, Fig. 5).

\section{Discussion}

The increase in the number of long-term survivors of OLT raises questions about the increasing number of patients who develop CKD and ESRD. In our study, $25.6 \%$ of 399 recipients developed CKD at 5 years after OLT, which was consistent with that in previous reports as described earlier in this article. The survival curve showed that post-OLT CKD was a strong predictor of patient prognosis, including mortality. The timedependent changes in renal function indicated that eGFR declined slowly year by year. It is important to identify 
Table 1 Perioperative characteristics of patients between the training set and validation set

\begin{tabular}{|c|c|c|c|}
\hline Characteristic & $\begin{array}{l}\text { Training set } \\
(n=293)\end{array}$ & $\begin{array}{l}\text { Validation set } \\
(n=106)\end{array}$ & $p$-value \\
\hline \multicolumn{4}{|l|}{ Demographic data } \\
\hline Age (years) & $52(46-59)$ & $51(44-58)$ & 0.27 \\
\hline Gender (N,\%) & & & 0.65 \\
\hline Female & $52(17.7 \%)$ & $16(15.1 \%)$ & \\
\hline Male & $241(82.3 \%)$ & $90(84.9 \%)$ & \\
\hline BMI $\left(\mathrm{Kg} / \mathrm{m}^{2}\right)$ & $24.22(22.32-26.40)$ & $23.69(21.11-26.61)$ & 0.40 \\
\hline MAP $(\mathrm{mmHg})$ & $92(85-98)$ & $91(84-97)$ & 0.96 \\
\hline \multicolumn{4}{|l|}{ Personal history } \\
\hline Smoking $(\mathrm{N}, \%)$ & $125(42.7 \%)$ & $53(50.0 \%)$ & 0.21 \\
\hline Alcoholism (N,\%) & $140(47.8 \%)$ & $52(49.1 \%)$ & 0.82 \\
\hline \multicolumn{4}{|l|}{ Pathogenesis of liver disease } \\
\hline HBV hepatitis $(\mathrm{N}, \%)$ & $229(78.2 \%)$ & $82(77.4 \%)$ & 0.89 \\
\hline Alcoholic liver cirrhosis (N,\%) & $21(7.2 \%)$ & $8(7.5 \%)$ & 1.00 \\
\hline Hepatocellular carcinoma $(\mathrm{N}, \%)$ & $139(47.4 \%)$ & $57(53.8 \%)$ & 0.31 \\
\hline Cholestatic disease $(\mathrm{N}, \%)$ & $19(6.5 \%)$ & $4(3.8 \%)$ & 0.47 \\
\hline \multicolumn{4}{|l|}{ Liver complications } \\
\hline Encephalopathy $(\mathrm{N}, \%)$ & $44(15.0 \%)$ & $11(10.4 \%)$ & 0.26 \\
\hline Ascites> $1 \mathrm{~L}(\mathrm{~N}, \%)$ & $25(23.6 \%)$ & $71(24.2 \%)$ & 1.00 \\
\hline \multicolumn{4}{|l|}{ Baseline medical status } \\
\hline Diabetes mellitus (N,\%) & $55(18.8 \%)$ & $21(19.8 \%)$ & 0.89 \\
\hline Hypertension $(\mathrm{N}, \%)$ & $38(13.0 \%)$ & $12(11.3 \%)$ & 0.73 \\
\hline Coronary heart disease $(\mathrm{N}, \%)$ & $8(2.7 \%)$ & $5(4.7 \%)$ & 0.34 \\
\hline Abdominal surgery history $(\mathrm{N}, \%)$ & $29(27.4 \%)$ & $62(21.2 \%)$ & 0.22 \\
\hline Preoperative LVEF (\%) & $63(61,65)$ & $62(60,64)$ & 0.10 \\
\hline Preoperative nonselective $\beta$ receptor blockers $(\mathrm{N}, \%)$ & $24(8.2 \%)$ & $7(6.6 \%)$ & 0.68 \\
\hline Preoperative diuretics $(\mathrm{N}, \%)$ & $110(37.5 \%)$ & $30(28.3 \%)$ & 0.10 \\
\hline \multicolumn{4}{|l|}{ Preoperative scores } \\
\hline MELD score & $12.94(9.50-18.87)$ & $12.59(9.50-17.00)$ & 0.31 \\
\hline Child-Pugh score & $10(8-14)$ & $9(8-11)$ & 0.14 \\
\hline \multicolumn{4}{|l|}{ Preoperative parameters } \\
\hline White blood cell $\left(\times 10^{9} / \mathrm{L}\right)$ & $3.39(2.28-4.91)$ & $3.61(2.26-5.33)$ & 0.53 \\
\hline Neutrophils $\left(\times 10^{9} / \mathrm{L}\right)$ & $2.28(1.46-3.51)$ & $2.43(1.58-3.62)$ & 0.57 \\
\hline Lymphocyte (× 109/L) & $0.67(0.40-1.03)$ & $0.65(0.47,1.11)$ & 0.44 \\
\hline Red blood cell (× 10 $12 / L)$ & $2.97(2.48-3.56)$ & $3.01(2.56-3.66)$ & 0.57 \\
\hline Hemoglobin (g/L) & $91(76-111)$ & $92(78-112)$ & 0.63 \\
\hline Platelet $\left(\times 10^{9} / \mathrm{L}\right)$ & $65(40-105)$ & $67(41-109)$ & 0.94 \\
\hline Total protein (g/L) & $59.00(55.00-62.93)$ & $59.62(54.21-64.80)$ & 0.56 \\
\hline Albumin (g/L) & $35.32(32.57-39.40)$ & 36.20 (32.20-39.02) & 0.97 \\
\hline TBIL $(\mu \mathrm{mol} / \mathrm{L})$ & $35.91(21.70-105.80)$ & $37.27(20.66-77.07)$ & 0.33 \\
\hline $\mathrm{DBIL}(\mu \mathrm{mol} / \mathrm{L})$ & $16.00(9.29-51.61)$ & $14.40(9.03-32.81)$ & 0.40 \\
\hline $\mid \mathrm{BIL}(\mu \mathrm{mol} / \mathrm{L})$ & $18.88(11.80-52.62)$ & $18.54(11.10-38.70)$ & 0.31 \\
\hline $\operatorname{ALT}(U / L)$ & $26.00(17.00-49.00)$ & $27.50(18.00-53.00)$ & 0.39 \\
\hline $\mathrm{AST}(\mathrm{U} / \mathrm{L})$ & $38.00(25.00-66.00)$ & $36.50(26.00-68.00)$ & 0.76 \\
\hline $\mathrm{LDH}(\mathrm{U} / \mathrm{L})$ & $159.00(133.00-193.00)$ & $158.00(139.00-202.00)$ & 0.53 \\
\hline $\mathrm{BUN}(\mathrm{mmol} / \mathrm{L})$ & $4.36(3.56-5.44)$ & $4.22(3.41-5.73)$ & 0.45 \\
\hline $\mathrm{sCr}(\mu \mathrm{mol} / \mathrm{L})$ & $60.00(48.00-74.00)$ & $65.00(54.00-78.00)$ & 0.01 \\
\hline $\mathrm{UA}(\mu \mathrm{mol} / \mathrm{L})$ & $231.00(179.00-293.00)$ & $247.00(176.00-314.00)$ & 0.29 \\
\hline $\mathrm{eGFR}\left(\mathrm{mL} / \mathrm{min} / 1.73 \mathrm{~m}^{2}\right)$ & $126.44(99.21-160.67)$ & $111.75(94.99,142.60)$ & 0.02 \\
\hline
\end{tabular}


Table 1 (continued)

\begin{tabular}{llll}
\hline Characteristic & $\begin{array}{l}\text { Training set } \\
(\boldsymbol{n}=\mathbf{2 9 3})\end{array}$ & $\begin{array}{l}\text { Validation set } \\
(\boldsymbol{n}=\mathbf{1 0 6})\end{array}$ & $\boldsymbol{p}$-value \\
\hline Postoperative parameters & & $991(679-1774)$ & 0.27 \\
Peak AST (U/L) & $1239(636-2325)$ & $16(5.5 \%)$ & 1.00 \\
Postoperative RRT (N,\%) & $5(4.7 \%)$ & $25(21,32)$ & 0.12 \\
Postoperative hospital stay (day) & $24(20,29)$ & $4(3-5)$ & 0.59 \\
Length of ICU stay (day) & $4(3-5)$ & $213(72.7 \%)$ & 0.26 \\
AKI & $71(67.0 \%)$ & $29(27.4 \%)$ & 0.90 \\
CKD & $78(26.6 \%)$ & & \\
\hline
\end{tabular}

Continuous variables are displayed as median and interquartile ranges

Abbreviations: BMI Body mass index, MAP Mean arterial pressure, LVEF Left ventricular ejection fraction, MELD Model for End-Stage Liver Disease, TBIL Serum total bilirubin, DBIL Serum direct bilirubin, IBIL Serum indirect bilirubin, ALT Alanine aminotransferase, AST Aspartate aminotransferase, LDH Lactate dehydrogenase, BUN Blood urea nitrogen, $s \mathrm{Cr}$ Serum creatinine, UA Uric acid, eGFR Estimated glomerular filtration rate, Peak AST AST peak value within first $24 \mathrm{~h}$ after OLT

Table 2 Characteristics of patients at 1-month after OLT between the training set and validation set

\begin{tabular}{|c|c|c|c|}
\hline Characteristic & $\begin{array}{l}\text { Training set } \\
(n=293)\end{array}$ & $\begin{array}{l}\text { Validation set } \\
(n=106)\end{array}$ & $p$-value \\
\hline White blood cell $\left(\times 10^{9} / \mathrm{L}\right)$ & $5.23(4.06-6.51)$ & $4.55(3.51-6.06)$ & 0.02 \\
\hline Neutrophils $\left(\times 10^{9} / \mathrm{L}\right)$ & $3.40(2.42-4.64)$ & $2.96(2.13-4.09)$ & 0.02 \\
\hline Lymphocyte $\left(\times 10^{9} / \mathrm{L}\right)$ & $0.97(0.63-1.39)$ & $0.93(0.65-1.29)$ & 0.93 \\
\hline Hemoglobin (g/L) & $114(102-126)$ & $113(99-125)$ & 0.70 \\
\hline Platelet $\left(\times 10^{9} / \mathrm{L}\right)$ & $167(109-253)$ & $156(103-232)$ & 0.41 \\
\hline NLR & $3.33(2.29-5.68)$ & $3.13(2.21-4.41)$ & 0.12 \\
\hline PLR & $150.99(111.34-252.34)$ & $165.71(106.17-238.22)$ & 0.78 \\
\hline$N / L P$ & $0.021(0.011-0.045)$ & $0.020(0.012-0.034)$ & 0.38 \\
\hline Total protein (g/L) & $65.68(59.52-70.14)$ & $65.16(60.40-70.01)$ & 0.81 \\
\hline Albumin (g/L) & $40.58(38.46-43.71)$ & 40.60 (37.49-44.08) & 0.73 \\
\hline TBIL $(\mu \mathrm{mol} / \mathrm{L})$ & $17.52(14.07-28.07)$ & $19.81(14.88-30.00)$ & 0.12 \\
\hline DBIL $(\mu \mathrm{mol} / \mathrm{L})$ & $8.32(5.84-13.66)$ & $9.50(6.50-15.50)$ & 0.12 \\
\hline $\mid \mathrm{BIL}(\mu \mathrm{mol} / \mathrm{L})$ & $9.30(7.16-13.40)$ & $10.40(7.71-14.74)$ & 0.19 \\
\hline $\operatorname{ALT}(U / L)$ & $28.00(16.00-53.00)$ & $27.00(15.00-56.00)$ & 0.60 \\
\hline AST (U/L) & $19.00(14.00-30.00)$ & $18.00(13.00-26.00)$ & 0.22 \\
\hline LDH (U/L) & $167.00(148.00-204.00)$ & $167.00(141.00-205.00)$ & 0.69 \\
\hline Glu (mmol/L) & $5.55(4.80-6.82)$ & $5.38(4.69-6.75)$ & 0.57 \\
\hline CysC (mg/L) & $1.17(1.01-1.47)$ & $1.18(1.01-1.39)$ & 0.66 \\
\hline C1q (mg/L) & 165 (139-198) & $171(142-199)$ & 0.46 \\
\hline BUN (mmol/L) & $6.17(4.85-7.62)$ & $5.82(4.74-7.51)$ & 0.43 \\
\hline $\mathrm{sCr}(\mu \mathrm{mol} / \mathrm{L})$ & $76.5(56.0-92.0)$ & $72.0(54.0,86.0)$ & 0.30 \\
\hline $\mathrm{UA}(\mu \mathrm{mol} / \mathrm{L})$ & $323.00(245.00-405.00)$ & $307.00(239.00-374.00)$ & 0.19 \\
\hline eGFR $\left(\mathrm{mL} / \mathrm{min} / 1.73 \mathrm{~m}^{2}\right)$ & $95.48(78.01-134.82)$ & $104.88(81.69-141.05)$ & 0.36 \\
\hline Average concentration of FK506 & $7.90(6.00-12.40)$ & $7.80(5.86-10.03)$ & 0.76 \\
\hline Average concentration of CsA & 707.55 (242.98-1219.70) & $369.95(261.80-949.25)$ & 0.43 \\
\hline Average concentration of Rapamycin & $2.75(2.72-427.35)$ & $1.68(1.66-3.74)$ & 0.40 \\
\hline
\end{tabular}

Continuous variables are displayed as median and interquartile ranges

Abbreviations: NLR Neutrophil lymphocyte ratio, PLR Platelet lymphocyte ratio, N/LP Ratio of Neutrophil to lymphocyte and platelet, TBIL Serum total bilirubin, $D B I L$ Serum direct bilirubin, IBIL Serum indirect bilirubin, ALT Alanine aminotransferase, AST Aspartate aminotransferase, LDH Lactate dehydrogenase, Glu Glucose, CysC Cystatin, C1q Complement C1q, BUN Blood urea nitrogen, sCr Serum creatinine, UA Uric acid, eGFR Estimated glomerular filtration rate, FK506 Tacrolimus, CsA Cyclosporin A 
Table 3 Characteristics of patients at 3-months after OLT between the training set and validation set

\begin{tabular}{|c|c|c|c|}
\hline Characteristic & $\begin{array}{l}\text { Training set } \\
(n=293)\end{array}$ & $\begin{array}{l}\text { Validation set } \\
(n=106)\end{array}$ & $p$-value \\
\hline Neutrophils $\left(\times 10^{9} / L\right)$ & $2.49(1.86-3.50)$ & $2.61(1.92-3.57)$ & 0.43 \\
\hline Lymphocyte (× 109/L) & $1.15(0.88-1.54)$ & $1.16(0.8-1.57)$ & 0.70 \\
\hline Hemoglobin (g/L) & $130(117-139)$ & 130 (119-141) & 0.64 \\
\hline Platelet $\left(\times 10^{9} / \mathrm{L}\right)$ & $114(90-175)$ & $132(93-186)$ & 0.11 \\
\hline NLR & $2.17(1.49-3.06)$ & $2.33(1.58-3.50)$ & 0.22 \\
\hline PLR & 102.99 (76.47-151.76) & $120.41(87.33-161.27)$ & 0.04 \\
\hline N/LP & $0.018(0.012-0.027)$ & $0.018(0.01-0.029)$ & 0.92 \\
\hline Albumin (g/L) & $41.60(37.41-44.70)$ & $42.80(39.50-45.42)$ & 0.02 \\
\hline TBIL ( $\mu \mathrm{mol} / \mathrm{L})$ & $15.26(11.33-19.49)$ & $15.30(11.08-21.25)$ & 0.88 \\
\hline DBIL $(\mu \mathrm{mol} / \mathrm{L})$ & $5.22(4.14-7.40)$ & $5.48(3.80-7.79)$ & 0.89 \\
\hline $\mathrm{ALT}(\mathrm{U} / \mathrm{L})$ & $21.00(15.00-37.00)$ & $22.00(14.00-36.00)$ & 0.95 \\
\hline AST (U/L) & $20.00(16.00-27.00)$ & $20.00(16.00-30.00)$ & 0.34 \\
\hline Glu (mmol/L) & $5.47(4.79-6.28)$ & $5.37(4.86-6.35)$ & 0.87 \\
\hline CysC (mg/L) & 1.135 (0.965-1.375) & $1.125(0.985-1.325)$ & 0.99 \\
\hline C1q (mg/L) & $168(139-198)$ & $171(145-194)$ & 0.74 \\
\hline BUN (mmol/L) & $5.90(4.91-7.58)$ & $5.97(4.94-6.97)$ & 0.70 \\
\hline $\mathrm{sCr}(\mu \mathrm{mol} / \mathrm{L})$ & 85.00 (66.00-93.00) & $80.00(60.00-93.00)$ & 0.19 \\
\hline UA ( $\mu \mathrm{mol} / \mathrm{L})$ & $359.00(305.00-439.00)$ & $356.00(292.00-416.00)$ & 0.38 \\
\hline $\mathrm{eGFR}\left(\mathrm{mL} / \mathrm{min} / 1.73 \mathrm{~m}^{2}\right)$ & $92.76(80.28-131.31)$ & $99.72(80.29-147.29)$ & 0.24 \\
\hline Average concentration of FK506 & $7.00(5.40-8.50)$ & $7.35(5.15-8.98)$ & 0.51 \\
\hline Average concentration of CsA & $390.60(246.25-575.35)$ & $121.28(73.68-478.19)$ & 0.09 \\
\hline Average concentration of Rapamycin & $4.14(3.59-5.12)$ & $3.97(2.92-4.51)$ & 0.33 \\
\hline
\end{tabular}

Continuous variables are displayed as median and interquartile ranges

Abbreviations: NLR Neutrophil lymphocyte ratio, PLR Platelet lymphocyte ratio, N/LP Ratio of Neutrophil to lymphocyte and platelet, TBIL Serum total bilirubin, $D B I L$ Serum direct bilirubin, IBIL Serum indirect bilirubin, ALT Alanine aminotransferase, AST Aspartate aminotransferase, $L D H$ Lactate dehydrogenase, Glu Glucose, CysC Cystatin, C1q Complement C1q, BUN Blood urea nitrogen, $s C r$ Serum creatinine, UA Uric acid, eGFR Estimated glomerular filtration rate, FK506 Tacrolimus, CsA Cyclosporin A

Table 4 Multivariable cox regression analysis for predictors of CKD

\begin{tabular}{lllll}
\hline Factors & $\boldsymbol{b}$ & $\boldsymbol{H R}$ & $\mathbf{9 5 \%} \mathbf{C l}$ & $\boldsymbol{P}$-value \\
\hline Age & 0.035 & 1.036 & $1.006-1.068$ & 0.018 \\
Female & 1.029 & 2.867 & $1.709-4.810$ & $<0.001$ \\
Hypertension & 0.479 & 1.670 & $0.962-2.898$ & 0.068 \\
eGFR before surgery & -0.004 & 0.996 & $0.991-1.001$ & 0.143 \\
Uric acid at 3 months & 0.002 & 1.002 & $1.001-1.004$ & 0.028 \\
Hemoglobin at 3 months & -0.031 & 0.970 & $0.956-0.983$ & $<0.001$ \\
Average concentration of & 0.002 & 1.002 & $1.001-1.003$ & $<0.001$ \\
CsA3 at 3 months & & & &
\end{tabular}

Abbreviations: $b$ Coefficient, $H R$ Hazard ratio, $C I$ Confidence interval, eGFR Estimated glomerular filtration rate, CSA Cyclosporin A

patients at risk for CKD, especially in the early course, to slow the occurrence and progression of CKD.

Although different studies have attempted to identify the best predictors of renal dysfunction after liver transplantation, a reliable and simple model to accurately predict CKD is still lacking. For this reason, we chose to construct and validate a monogram model based on Cox regression analysis. A nomogram model can integrate multiple factors, enabling clinicians to comprehensively predict the prognosis of a patient and make clinical decisions. The model developed in our study integrated patients' age at surgery, female sex, preoperative hypertension, baseline eGFR, UA, $\mathrm{Hb}$, and average plasma concentration of CsA at 3 months after OLT as predictors of the development of CKD.

The first predictor in our model was female sex, which has previously been recognized as a risk factor for CKD. Female patients suffered a faster decline in renal function, as illustrated by the plot of the decrease in eGFR between males and females in this study. In contrast, among nontransplant patients, male sex was associated with a faster decline in eGFR. Female sex is also a risk factor for CKD after heart, lung, and kidney transplantation. The exact mechanism of this association is not clear. A possible explanation may be that females may be more susceptible to CNI-mediated renal injury [21]. 


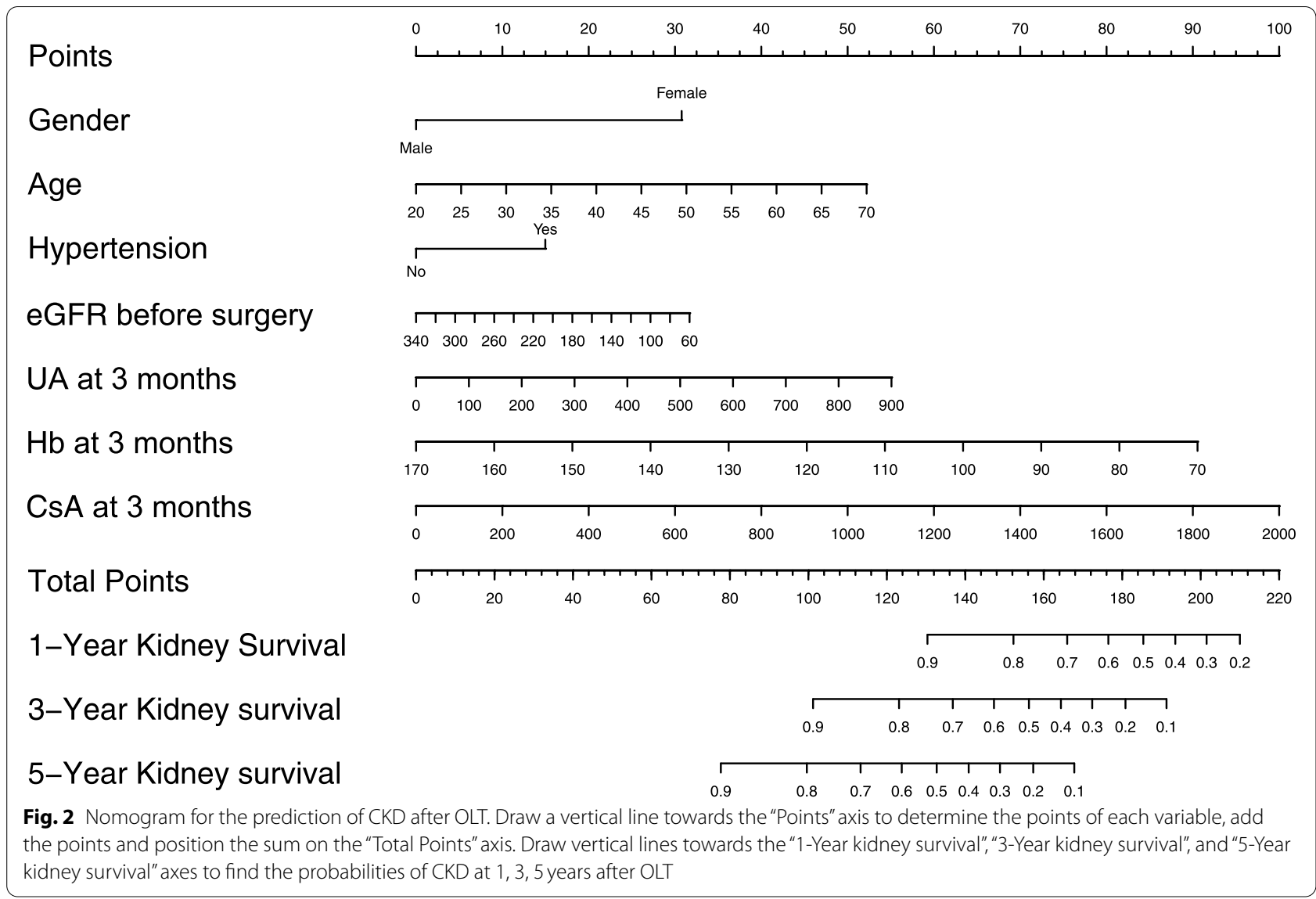

Recipients with hypertension and low eGFR are more prone to suffering IRI during and after surgery due to trauma and circulation instability [6]. Although their $p$ values were $>0.05$, our nomogram model showed that preoperative hypertension and low eGFR were important predictors of new-onset CKD after OLT.

In our population study, the UA level at 3 months after OLT was found to be a risk factor for new-onset CKD. Elevated serum levels of UA are associated with the onset and progression of CKD in many populations, such as the general population [22], patients with hypertension [23], and kidney transplantation patients [24]. Although hyperuricaemia is common after OLT, its association with the development of CKD has not been well described. It was reported that post-OLT hyperuricaemia was related to immunosuppressants, primarily CNIs (CsA and FK506), which decrease UA excretion by reducing GFR and increasing UA reabsorption in renal tubules. The incidence of hyperuricaemia was much higher in recipients treated with CsA than in those treated with FK506 in some reports. Multiple mechanisms are related to renal damage caused by UA, including contributions to the development of hypertension [25], activation of the renin-angiotensin-aldosterone system (RAAS) [26-28], inflammation, and oxidative stress reactions [29]. In patients with CKD, a high level of serum UA was associated with cardiovascular diseases [30], a higher risk of incident RRT, and all-cause mortality [31]. It is important to reduce UA levels in OLT recipients to prevent the occurrence of CKD and improve prognosis. The treatments include changing the unhealthy lifestyle of patients and using drugs such as febuxostat, allopurinol, and topiroxostat. In addition, CNIs should be avoided, or the dose should be reduced as much as possible. Some reports have shown that the reduction or even withdrawal of CNIs after OLT combined with MMF or mTOR inhibitors can contribute to the reduction in UA levels $[32,33]$. Therefore, serum UA levels should be closely monitored by clinicians during follow-up. Immunosuppression programs should be individualized according to the specific situation of the recipient. Due to its negative impact on prognosis, serum levels of uric acid were monitored and intervened carefully for all patients in our centre. The HR of UA at 3 months was low in this study, which might be related to the intervention of lowering UA during follow-up.

Another risk factor for CKD after OLT was low $\mathrm{Hb}$ levels at 3 months. The association between 


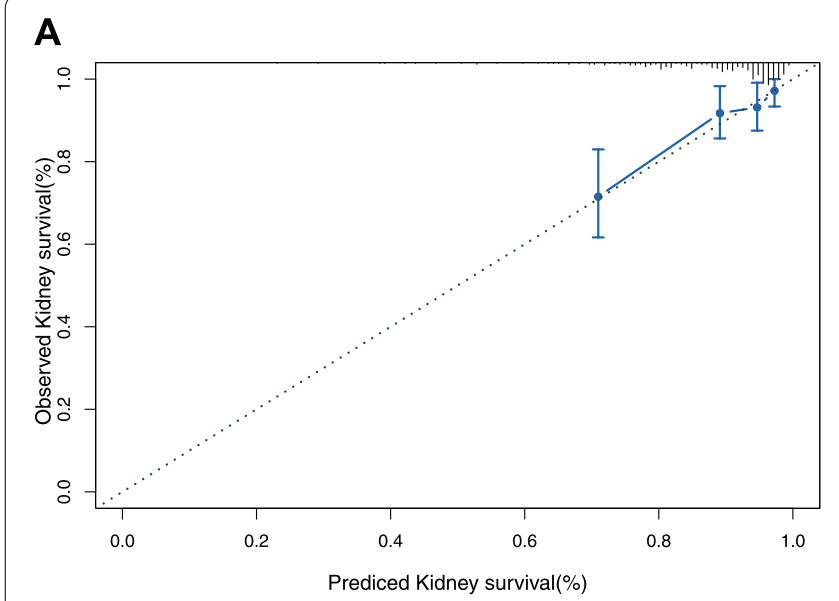

\section{B}

C
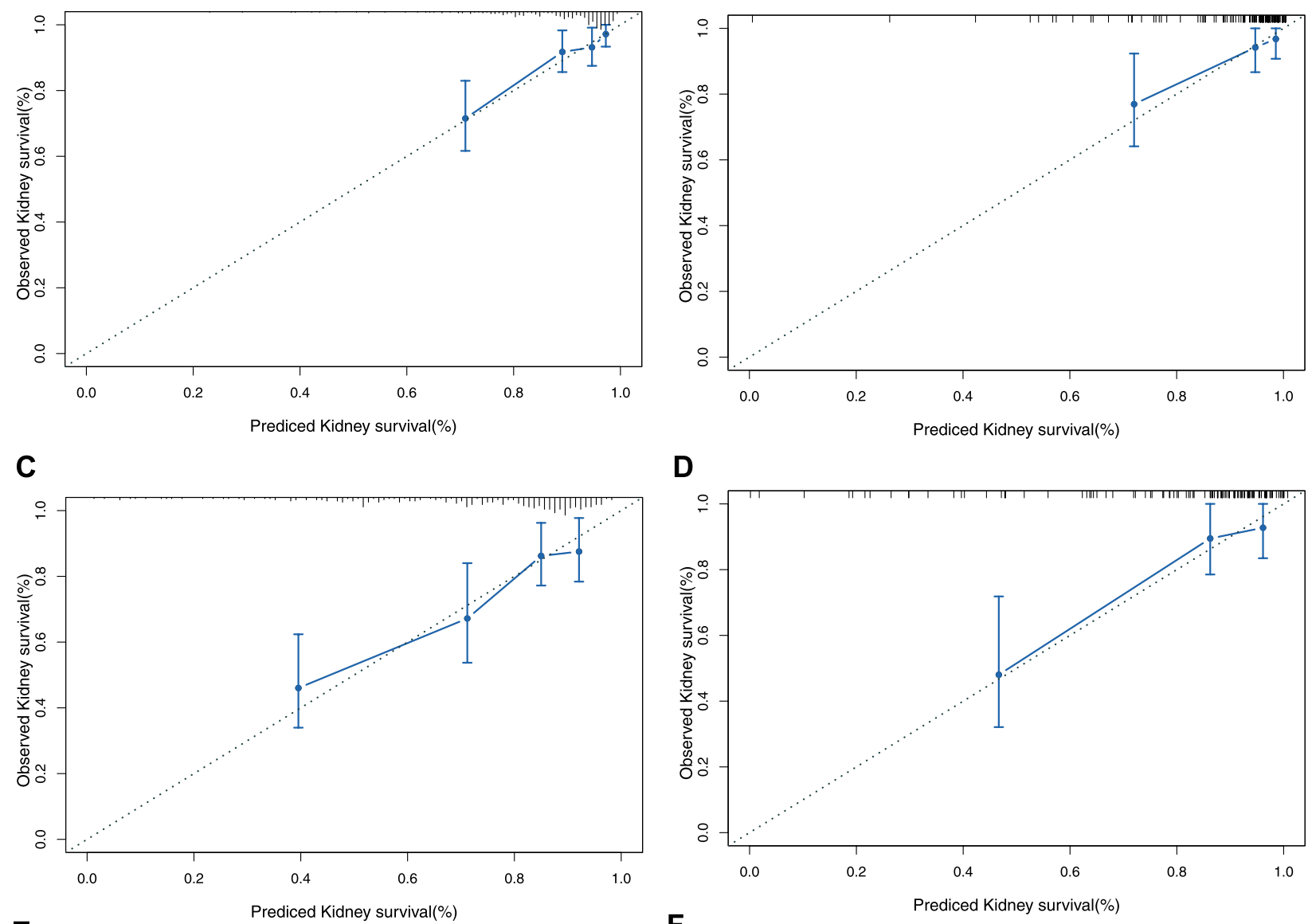

D

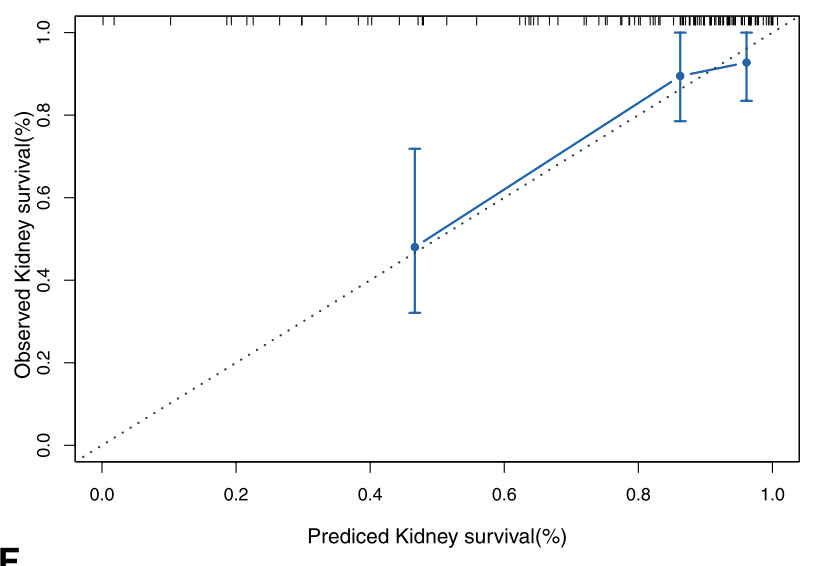

E

$\mathbf{F}$
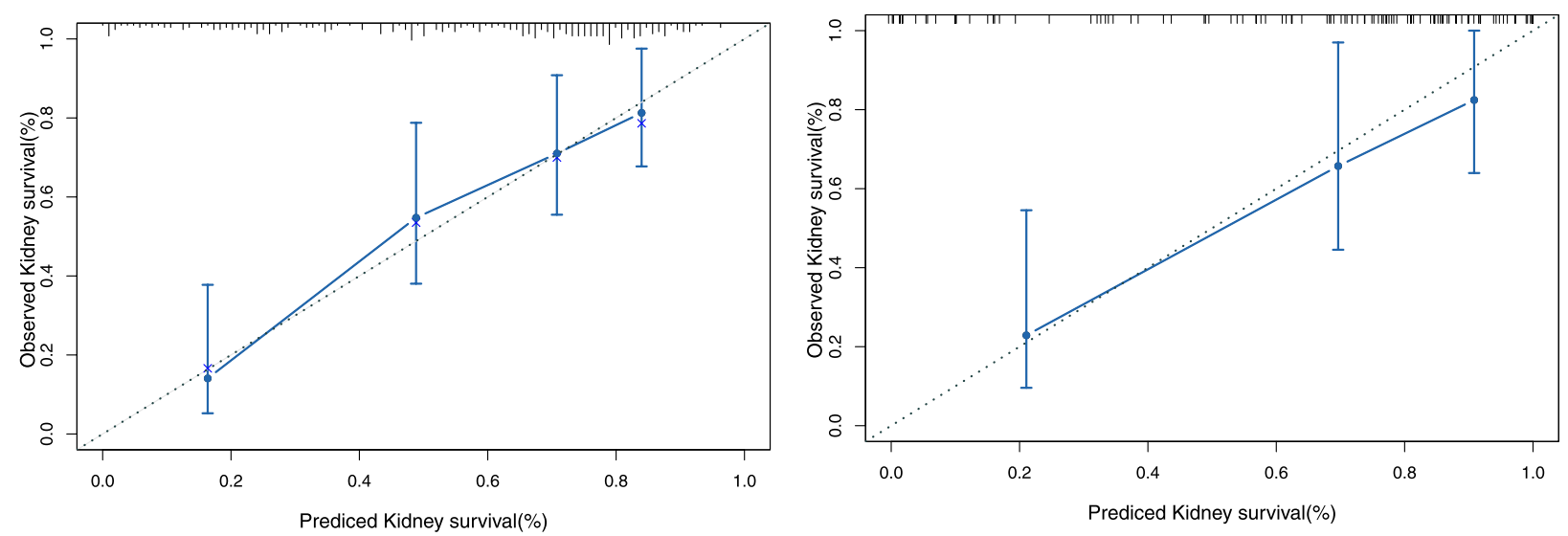

Fig. 3 Calibration plot of the probabilities of CKD predicted by the nomogram model vs. the observed probabilities. Calibration at 1 year in the training set $(\mathbf{A})$ and validation set $(\mathbf{B})$. Calibration at 3 years in the training set $(\mathbf{C})$ and validation set $(\mathbf{D})$. Calibration at 5 years in the training set $(\mathbf{E})$ and validation set $(\mathbf{F})($ all $p>0.05)$

preoperative $\mathrm{Hb}$ and CKD after OLT was found in a previous Chinese cohort study [6]. In our study, low $\mathrm{Hb}$ at 3 months after OLT may contribute to CKD by reducing the oxygen capacity of the blood, enhancing oxidative stress, and impairing haemostasis. In addition, a recent study from Korea revealed that CKD patients with anaemia are at high risk for hyperuricaemia, which may further promote the development of CKD. The reason for the decrease in $\mathrm{Hb}$ at 3 months may be related to the use of immunosuppressive medications. 
A

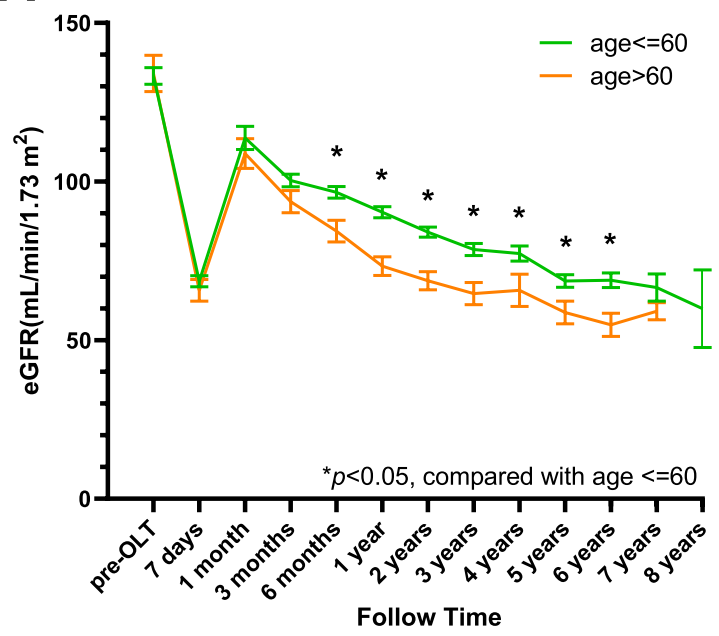

C

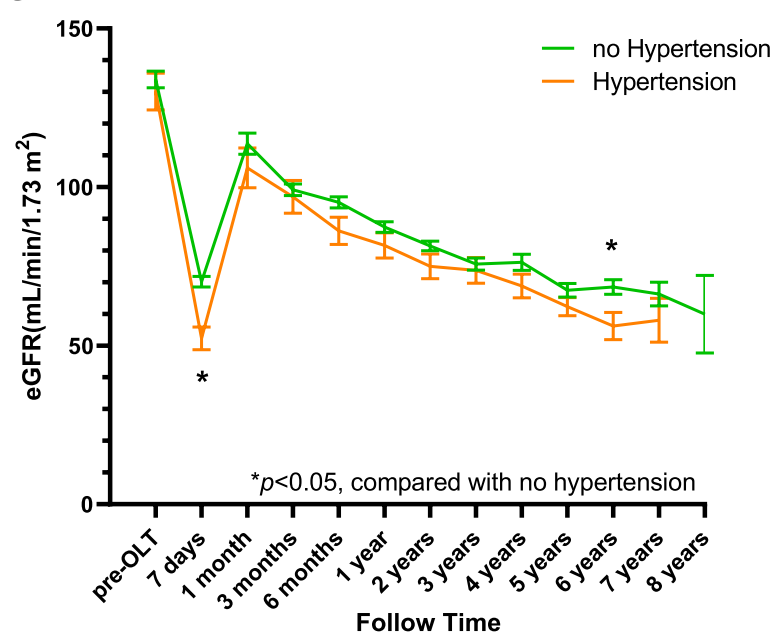

E

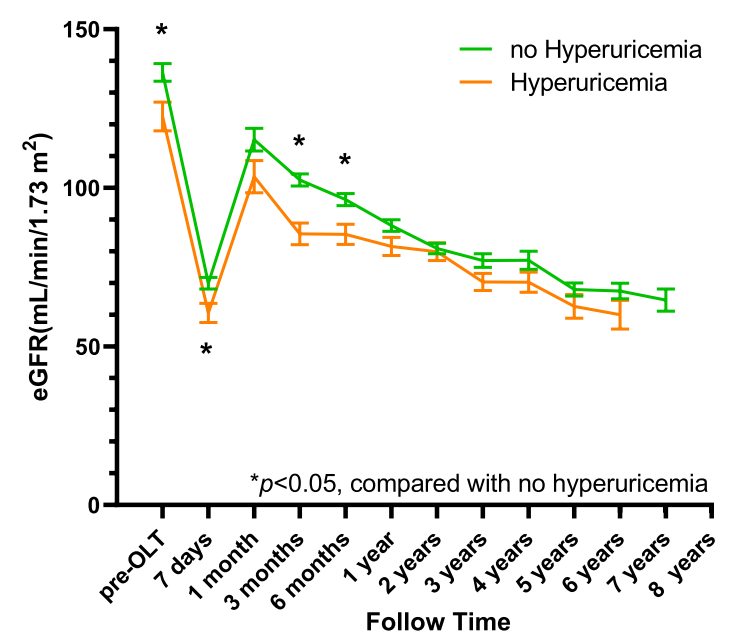

B

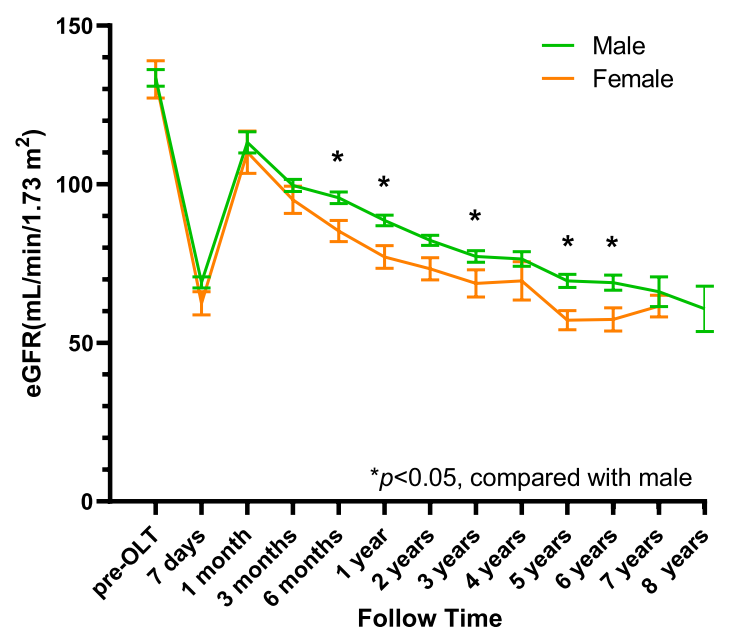

D

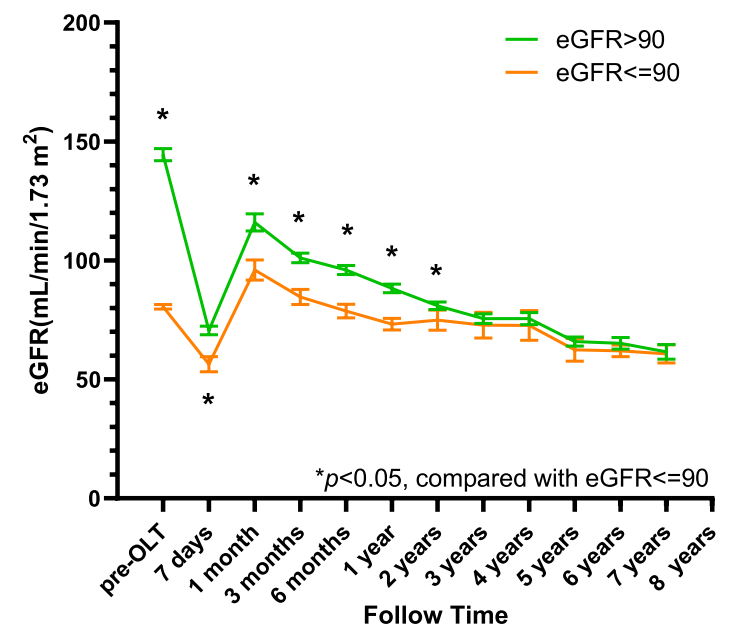

$\mathbf{F}$

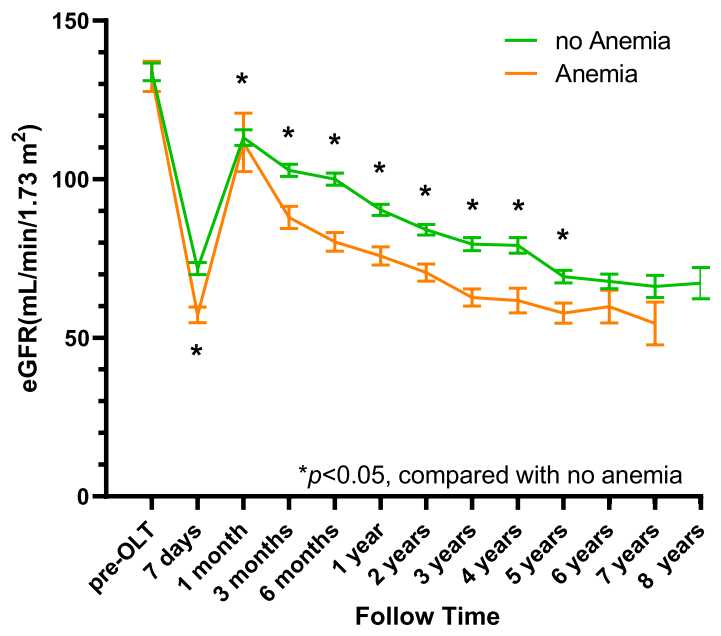

Fig. 4 Time-dependent changes in renal function according to the predictors in the multivariable Cox regression analysis. ${ }^{*} p<0.05$, compared with the reference group (nonparametric test) 


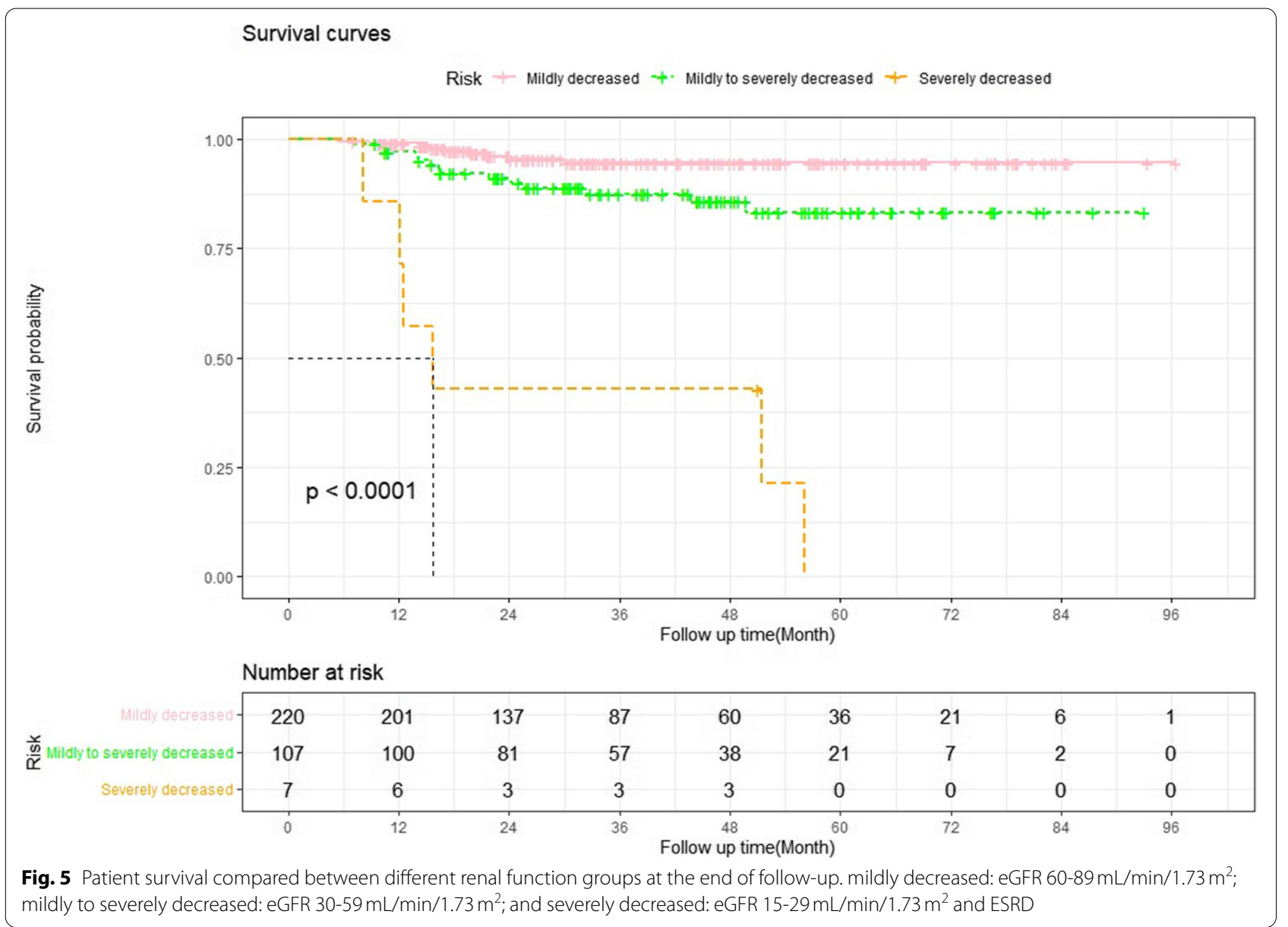

MMF, azathioprine, and sirolimus are common causes of bone marrow suppression, which thus leads to a decrease in Hb after OLT [34].

It is well known that the dose-dependent nephrotoxicity of CNIs also plays an important role in the occurrence of new-onset CKD. In our study, the average plasma concentration of CsA at 3 months was an independent risk factor for the development of postOLT CKD. Calcineurin stimulates vascular endothelial cells to secrete endothelin, release angiotensin II and overexpress transforming growth factor- $\beta$. This process is accompanied by a decrease in stromal degrading enzyme activity, leading to the hyperconstruction of glomerular arterioles, hyalinosis, chronic thromboembolism, and the excessive synthesis of extracellular matrix. Finally, it results in tubule atrophy and interstitial fibrosis and reduces renal blood flow and glomerular filtration [6]. However, the concentration of tacrolimus was not included in the nomogram model. Most of our recipients were on long-term immunosuppression with tacrolimus, and interestingly, CKD recipients had significantly lower levels of tacrolimus.
This finding was consistent with the finding of some previous studies, which did not show a correlation between tacrolimus levels and the development of CKD [35]. This may be due to the adjustment of tacrolimus dose in patients with known renal impairment in our centre.

Finally, a nomogram model was constructed based on multivariable Cox regression analysis, which provides clinicians with a visual tool to understand the impact of predictors on renal function after OLT. In addition, by comprehensively analysing all predictors included, we can accurately calculate the probabilities of CKD for each recipient, making the results more personalized. After evaluation, the $\mathrm{C}$-index of the nomogram model was 0.75 for the training set and 0.80 for the validation set, indicating that the nomogram model has a strong ability to distinguish patients with and without CKD. The calibration curves in the training set and validation set showed that the predicted probabilities of CKD at 1,3 , and 5 years after OLT were consistent with the observed probabilities, which indicates the accurate prediction ability of our model. 
Several prediction models for CKD after OLT have been developed. A CKD prediction formula was developed from New York based on urinary neutrophil gelatinase-associated lipocalin (uNGAL) at $24 \mathrm{~h}$ after OLT as the most important risk factor. However, uNGAL-24h is not routinely examined in other centres. Moreover, this prediction model has not been validated in terms of its calibration abilities [36]. Recently, a biomarker model predictive of renal outcomes after liver transplantation was constructed based on a large population from multiple centres. The levels of $\beta 2$-microglobulin and CD40 antigen included in the prediction model are not routinely examined in other centres. Despite the high area under the curve (AUC), no calibration verification was performed [37]. The pocket guide to identifying patients at risk for CKD after liver transplantation, which includes hepatitis $C$ virus $(\mathrm{HCV})$ as one of the predictive factors, is a reliable prediction model for German patients. However, it does not apply to Chinese patients because HCV is more common in Western countries than in China [38]. Another model from Italy has been validated through AUC calculation and calibration; however, the sample size was relatively small, and there was no visual tool developed for the model [2]. In addition, none of these prediction models have been used in Chinese recipients.

Compared with these models, our model has the following advantages. First, it is the first nomogram model constructed based on OLT recipients for predicting CKD. Second, the predictors included in our model were readily available at surgery or routinely tested in follow-up, which is conducive to the popularization of the model. Third, our model had excellent discrimination and calibration abilities in the training set and validation set. It is a simple and reliable tool to distinguish recipients at high risk for CKD after OLT at 1, 3, and 5 years. In addition, this model can be used earlier at 3 months after OLT, assisting clinicians in adjusting immunosuppressive drugs in advance.

This study had several limitations. First, this study had a retrospective single-centre design, and the sample size was small, so the model should be evaluated prospectively in other large multi-centre studies to demonstrate its applicability. Second, the use of MDRD to assess renal function may lack accuracy in candidates awaiting liver transplantation, possibly because of reduced muscle mass and/or hyperbilirubinemia. It would overestimate renal function in patients with poor function (GFR $<40 \mathrm{~mL} / \mathrm{min}$ ) and underestimate renal function in patients with reasonable renal function (GFR $>40 \mathrm{~mL} / \mathrm{min}$ ) [17]. However, this is the most frequently used method to estimate kidney function.
Third, due to data deficiency, we did not analyse the relationship between urinalysis data such as proteinuria and postoperative CKD. Fourth, to more accurately clarify the impact of CKD on the long-term prognosis after OLT, it is necessary to conduct a longer follow-up study.

In conclusion, the renal function of most recipients decreased and recovered rapidly in the first week after OLT and then decreased slowly year by year. Patients with severe CKD had a poor survival prognosis. We constructed a nomogram model for predicting post-OLT CKD for the first time. With excellent discrimination and calibration, this nomogram model can accurately predict patients at high risk for CKD after OLT. Therefore, we can take measures to prevent or slow the development and progression of CKD in advance, such as reducing UA levels, improving $\mathrm{Hb}$ levels, and withdrawing or minimizing the use of CNIs in follow-up.

\section{Abbreviations}

AKI: Acute kidney injury; AST: Aspartate aminotransferase; ALT: Alanine transaminase; AIC: Akaike's information criterion; BMI: Body mass index; BUN: Blood urea nitrogen; CKD: Chronic kidney disease; CKD-MDRD: the Modification of Diet in Renal Disease equation; CysC: Cystatin; C1q: Complement C1q; CsA: Cyclosporin A; Cl: Confidence interval; DBIL: Serum direct bilirubin; eGFR: Estimated glomerular filtration rate; ESRD: End-stage renal disease; FK506:

Tacrolimus; Glu: Glucose; HR: Hazard ratio; IQR: Interquartile range; IRI: Ischemia reperfusion injury; IBIL: Serum indirect bilirubin; KDIGO: Kidney Disease Improving Global Outcomes; LVEF: Left ventricular ejection fraction; LDH: Lactate dehydrogenase; MAP: Mean arterial pressure; MELD: Model for End-Stage Liver Disease; NLR: Neutrophil lymphocyte ratio; N/LP: Ratio of Neutrophil to lymphocyte and platelet; OLT: Orthotopic liver transplantation; POD: Postoperative day; Peak AST: AST peak value within first $24 \mathrm{~h}$ after OLT; PLR: Platelet lymphocyte ratio; RRT: Renal replacement therapy; sCr: Plasma creatinine; TBIL: Serum total bilirubin; UA: Uric acid.

\section{Acknowledgments}

Not applicable.

\section{Authors' contributions}

Dandan Guo participated in study design, data collection, data analysis, and manuscript draft. Huifang Wang, Jun Liu are contributed to the performance of research and modification of the draft. Hang Liu and Ming Zhang participated in the performance of research and contributed analytic tools. Zixuan Fu was an independent member who collected the data. Xuemei Liu is the senior author of this manuscript and contributed to the study design, implementation, manuscript draft, and critical revision of the article. All authors read and approved the final manuscript.

\section{Funding}

This work was supported by the Project of Science and Technology of Qingdao People's Livelihood under Grant number 19-6-1-18-nsh.

\section{Availability of data and materials}

The datasets used and/or analyzed during the current study are available from the corresponding author on reasonable request.

\section{Declarations}

\section{Ethics approval and consent to participate}

The study was conducted according to the principles of the Declaration of Helsinki and approved by the Medical Ethics Committee of The Affiliated Hospital of Qingdao University with informed consent of all subjects and/ 
or their legal guardian(s) (the ethics approval number is QYFY WZLL 26283). All methods were performed in accordance with the relevant guidelines and regulations.

\section{Consent for publication}

Not applicable.

\section{Competing interests}

The authors report no conflict of interest.

Received: 14 August 2021 Accepted: 15 December 2021

Published online: 16 January 2022

\section{References}

1. Kim WR, Smith JM, Skeans MA, et al. OPTN/SRTR 2012 annual data report: liver. Am J Transplant. 2014;14(Suppl 1):69-96.

2. Giusto M, Berenguer M, Merkel C, et al. Chronic kidney disease after liver transplantation: pretransplantation risk factors and predictors during follow-up. Transplantation. 2013;95:1148-53.

3. Weber ML, Ibrahim HN, Lake JR. Renal dysfunction in liver transplant recipients: evaluation of the critical issues. Liver Transpl. 2012:18:1290-301.

4. Hao JC, Wang WT, Yan LN, et al. Effect of low-dose tacrolimus with mycophenolate mofetil on renal function following liver transplantation. World J Gastroenterol. 2014;20:11356-62.

5. Maurel P, Prémaud A, Carrier P, et al. Evaluation of longitudinal exposure to tacrolimus as a risk factor of chronic kidney disease occurrence within the first year post-liver transplantation. Transplantation. 2020;105:1585-94.

6. Li Y, Li B, Wang W, et al. Risk factors for new-onset chronic kidney disease in patients who have received a liver transplant. Exp Ther Med. 2018;15:3589-95.

7. Cantarovich M, Tchervenkov J, Paraskevas S, et al. Early changes in kidney function predict long-term chronic kidney disease and mortality in patients after liver transplantation. Transplantation. 2011;92:1358-63.

8. Bahirwani R, Reddy KR. Outcomes after liver transplantation: chronic kidney disease. Liver Transpl. 2009;15(Suppl 2):S70-4.

9. Peng JC, Li YJ, Wang J, et al. Incidence of chronic kidney disease after orthotopic liver transplantation in a Chinese cohort. Clin Exp Nephrol. 2020;24:806-12.

10. Lamattina JC, Foley DP, Mezrich JD, et al. Chronic kidney disease stage progression in liver transplant recipients. Clin J Am Soc Nephrol. 2011;6:1851-7.

11. Bahirwani $R$, Forde $K A, M u Y$, et al. End-stage renal disease after liver transplantation in patients with pre-transplant chronic kidney disease. Clin Transpl. 2014;28:205-10.

12. Kang GW, Lee IH, Ahn KS, et al. One-year follow-up of the changes in renal function after liver transplantation in patients without chronic kidney disease. Transplant Proc. 2016;48:1190-3.

13. Campo A. Chronic renal failure after transplantation of a nonrenal organ. N Engl J Med. 2003;349:2563-5.

14. Hilmi IA, Damian D, Al-Khafaji A, et al. Acute kidney injury following orthotopic liver transplantation: incidence, risk factors, and effects on patient and graft outcomes. Br J Anaesth. 2015;114:919-26.

15. Leithead JA, Ferguson JW, Bates CM, et al. Chronic kidney disease after liver transplantation for acute liver failure is not associated with perioperative renal dysfunction. Am J Transplant. 2011;11:1905-15.

16. Rahman S, Davidson BR, Mallett SV. Early acute kidney injury after liver transplantation: predisposing factors and clinical implications. World J Hepatol. 2017;9(18):823-32.

17. Gonwa TA, Jennings L, Mai ML, Stark PC, Levey AS, Klintmalm GB. Estimation of glomerular filtration rates before and after orthotopic liver transplantation: evaluation of current equations. Liver Transpl. 2004; 10(2):301-9.

18. National Kidney Foundation. K/DOQI clinical practice guidelines for chronic kidney disease: evaluation, classification, and stratification. Am J Kidney Dis. 2002;39(2 Suppl 1).

19. KDIGO AKI Work Group. Kidney Disease: Improving Global Outcomes (KDIGO) CKD work group KDIGO 2012 clinical practice guideline for the evaluation and management of chronic kidney disease. Kidney Int Suppl. 2013:3:1-150.

20. Yap W-K, Shih M-C, Kuo C, et al. Development and validation of a nomogram for assessing survival in patients with metastatic lung cancer referred for radiotherapy for bone metastases. JAMA Netw Open. 2018;1:e183242.

21. Leithead JA, Ferguson JW, Hayes PC. Modifiable patient factors are associated with the late decline in renal function following liver transplantation. Clin Transpl. 2012;26:E316-23.

22. Tada K, Maeda T, Takahashi K, et al. Association between serum uric acid and new onset and progression of chronic kidney disease in a Japanese general population: Iki epidemiological study of atherosclerosis and chronic kidney disease. Clin Exp Nephrol. 2021;25:751-9.

23. Kaewput W, Thongprayoon C, Rangsin R, et al. Association between serum uric acid and chronic kidney disease in patients with hypertension: a multicenter nationwide cross-sectional study. J Evid Based Med. 2019;12:235-42.

24. Yang $\mathrm{H}, \mathrm{Chen} \mathrm{Q}$, Huang $\mathrm{A}$, et al. The impact of hyperuricemia on longterm clinical outcomes of renal transplant recipients: a systematic review and meta-analysis. J Pharm Pharm Sci. 2021;24:292-307.

25. Feig DI, Madero M, Jalal DI, et al. Uric acid and the origins of hypertension. J Pediatr. 2013;162:896-902.

26. Mazzali M, Hughes J, Kim YG, et al. Elevated uric acid increases blood pressure in the rat by a novel crystal-independent mechanism. Hypertension. 2001;38(5):1101-6.

27. Xu C, Lu A, Lu X, et al. Activation of renal (pro)renin receptor contributes to high fructose-induced salt sensitivity. Hypertension. 2017;69(2):339-48.

28. Eräranta A, Kurra V, Tahvanainen AM, et al. Oxonic acid-induced hyperuricemia elevates plasma aldosterone in experimental renal insufficiency. J Hypertens. 2008;26(8):1661-8.

29. Isaka Y, Takabatake Y, Takahashi A, Saitoh T, Yoshimori T. Hyperuricemia-induced inflammasome and kidney diseases. Nephrol Dial Transplant. 2016;31(6):890-6.

30. Chiu T-H, Wu P-Y, Huang J-C, et al. Hyperuricemia is associated with left ventricular dysfunction and inappropriate left ventricular mass in chronic kidney disease. Diagnostics. 2020;10:514.

31. Lee C-L, Wang J-S. Effects of hyperuricemia on incident renal replacement therapy and all-cause mortality among patients with chronic kidney disease stages 3-5: a retrospective cohort study. Sao Paulo medical journal $=$. Rev Paul Med. 2019;137:523-9.

32. Pons JA, Ramírez P, Revilla-Nuin B, et al. Immunosuppression withdrawal improves long-term metabolic parameters, cardiovascular risk factors and renal function in liver transplant patients. Clin Transpl. 2009;23:329-36.

33. Malheiro J, Almeida M, Fonseca l, et al. Hyperuricemia in adult renal allograft recipients: prevalence and predictors. Transplant Proc. 2012:44:2369-72.

34. Maheshwari A, Mishra R, Thuluvath PJ. Post-liver-transplant anemia: etiology and management. Liver Transpl. 2004;10(2):165-73.

35. Kalisvaart M, Schlegel A, Trivedi PJ, et al. Chronic kidney disease after liver transplantation: impact of extended criteria grafts. Liver Transpl. 2019;25:922-33.

36. Cullaro G, Pisa JF, Brown RS, et al. Early postoperative neutrophil gelatinase-associated lipocalin predicts the development of chronic kidney disease after liver transplantation. Transplantation. 2018;102:809-15.

37. Levitsky J, Asrani SK, Klintmalm G, et al. Discovery and validation of a biomarker model (PRESERVE) predictive of renal outcomes after liver transplantation. Hepatology. 2020;71:1775-86.

38. Weismüller TJ, Lerch C, Evangelidou E, et al. A pocket guide to identify patients at risk for chronic kidney disease after liver transplantation. Transpl Int. 2015:28:519-28.

\section{Publisher's Note}

Springer Nature remains neutral with regard to jurisdictional claims in published maps and institutional affiliations. 